\title{
An Associated Degree in Electronic Engineering with specialization in optics
}

J. Sánchez-Mondragón, J. Escobedo-Alatorre, M. Tecpoyotl-Torres, M. Basurto-Pensado, R. RodriguezVera, et al.

J. J. Sánchez-Mondragón, J. Escobedo-Alatorre, M. Tecpoyotl-Torres, M. A. Basurto-Pensado, R. Rodriguez-Vera, R. Selvas, "An Associated Degree in Electronic Engineering with specialization in optics," Proc. SPIE 9663, Eighth International Topical Meeting on Education and Training in Optics and Photonics, 96632T (6 October 2003); doi: 10.1117/12.2208445

SPIE Event: Eighth International Topical Meeting on Education and Training in Optics and Photonics, 2003, Tucson, Arizona, United States 


\title{
An Associated Degree in Electronic Engineering with specialization in optics
}

\author{
J.J. Sánchez-Mondragón \\ Instituto Nacional de Astrofisica, Óptica y Electrónica \\ Luis Enrique Erro \#1, Apdo. 51 y 216 CP 72000, Tonantzintla, Puebla, México \\ telephone: 522222 128583; fax: 522222 472231; e-mail: jsanchez@inaoep.mx
}

\author{
J. Escobedo-Alatorre, M. Tecpoyotl-Torres, and M.A. Basurto-Pensado \\ Centro de Investigación en Ingeniería y Ciencias Aplicadas \\ Av. Universidad 1001, Col. Chamilpa, Cuernavaca, Morelos, México
}

\section{R. Rodríguez-Vera and R. Selvas}

Centro de Investigación en Optica

Lomas del Bosque 115, Colonia Lomas del Campestre, León, Guanajuato, México

\begin{abstract}
We have experienced an Associated Degree on Engineering and a subsequent Bs on Electronic Engineering that uses Optics as the essential introduction to Modern Ondulatory Physics. This first experience in Mexico is important because the popular origin of Mexican Engineering Students, that represents the $27 \%$ of the National enrollment [1].

(C)2003 Optical Society of America

OCIS codes: (000.2060) Education, (000,2170) Equipment and techniques
\end{abstract}

\section{Introduction}

An Associated Degree on Engineering (ADE) emerges from the necessities of a developing country. The current industry also requires personal with specialization in specific areas. In particular optics is growing within the context of other engineering such as electronics. In this context, it is required to provide opportunities to students who want to complete a studies program, which covers technical and engineering basis. An ADE and the subsequent Bs on Electronic Engineering have then been created for this necessity. Chronologically these are typically composed by a plan of 4 years degree divided in two parts, where students covering their first two years, they have the opportunity to explore all the technical issues of an Electronics degree. Subsequently, the experienced students are ready to incorporate straightforward to the demanding industry. On the other hand, while the student is deciding for incorporating to the industry, the plan gives the flexible option to continue and finish a Bs degree in the subsequence years.

The basic education in Mexico has typically a framework where optics raises from physicist and electronics from Engineering. However, a program with both specialties claims many challenges and benefits, such as, how to incorporate photonics, optical engineering, quantum mechanics and electronics in a unified program.

This vision was widely accepted at the UAEM. In 1998, a syllabus of the Electrical engineering was proposed, so that it fulfilled the requirements of an $\mathrm{ADE}$ in electronics engineering with specialization in optics, one of the first of its kind implemented in Mexico. This paper will be giving the experience concerning in the development of such a plan of ADE \& Bs.Eng. With 5 years of experience in this program, many challenges have been treated and overcome.

\section{Summary}

With an invitation from the Morelos State University (UAEM), we started in the creation for a research centre in electrical engineering. The main objective was to support the engineering program already inscribed at the UAEM as well as to open the electronic and computer areas with a new scheme.

A survey data [1] by the Mexican ministry of education revealed that up to $80 \%$ of student leave the university system because social-economic problems. For that reason, a reorganization was claimed for solve all these problems, creating a challenging and interesting academic problem.

The ADE and the Bs en Electronic engineer were then created and this comprise of two stages schedule. The first step has a duration of $2 \frac{1}{2}$ years and has a design in such a way that enables students to focus on practical abilities and the required physics-mathematics background. The scheme allows to incorporate the students directly to the productive sectors at the end of this first step, and creates the economical conditions such that a larger population continue the second stage and to conclude with an engineering degree. 
From a methodological point of view, the engineering degrees in Mexico did not have a strong emphasis on physics and mathematics. However, this new program shows the students the convenience of learning optics because of current availability of optoelectronic components, and by doing it, eases the understanding of quantum mechanics that has traditionally limited their perusing graduate studies.

It is important to point out that these programs were developed pursuing the ABET guidelines (Accreditation Board for Engineering and Technology), that almost simultaneously published the ADE guidelines. The program has received positive remarks from the department of Education and the General Direction of Technological Universities, responsible of the ADE programs in Mexico.

\section{References}

[1] “Programa Nacional de Educación Superior 2000’2006”, Reporte de la SEP, (2000). 